\title{
DETERMINANTES SOCIAIS DA SAÚDE: O INSTITUÍDO CONSTITUCIONAL NO SISTEMA ÚNICO DE SAÚDE
}

\author{
Social Determinants of Health: The Constitutional Instituted in the Unified Health \\ System
}

\section{Determinantes sociales de la salud: el instituido constitucional del sistema único de salud}

Kelen Gomes Ribeiro

Universidade Federal do Ceará - UFC - Fortaleza (CE) - Brasil

\author{
Jaina Bezerra de Aguiar \\ Universidade Estadual do Ceará - UECE - Fortaleza (CE) - Brasil
}

\author{
Luiz Odorico Monteiro de Andrade \\ Fundação Oswaldo Cruz - FIOCRUZ - Fortaleza (CE) - Brasil \\ Universidade Federal do Ceará - UFC - Fortaleza (CE) - Brasil
}

\section{RESUMO}

Objetivo: Compreender a relação entre o instituído constitucional sobre os determinantes sociais da saúde e a vivência urbana num território em situação de vulnerabilidade social. Métodos: Baseou-se na abordagem qualitativa, com utilização de grupo focal e de entrevista semi estruturada, aplicados a 45 participantes. Realizou-se análise de conteúdo, o que possibilitou a categorização dos dados a partir da técnica de análise temática. Resultados: Os resultados mostraram a vivência no território com insuficiência na oferta de serviços que determinam a saúde da população, apontando para desafios marcantes como a baixa cobertura na Atenção Primária à Saúde e a violência urbana. A busca por qualidade da assistência no Sistema Único de Saúde apresentou-se como estratégia de resistência. Apontou-se para a necessidade de valorização do território como lócus de cuidado das pessoas, com ações intersetoriais que visem promover a saúde da população. Conclusão: Reconhecem-se especificidades dos determinantes sociais na saúde de populações que apresentam condições de vida permeadas por iniquidades e expõem-se subsídios para elaborar medidas que contribuam para superação desses quadros, de forma a gerar equidade social.

Descritores: Determinantes Sociais da Saúde; Sistema Único de Saúde; Promoção da Saúde.

\begin{abstract}
Objective: To understand the relationship between what the Brazilian Constitution established about the social determinants of health and urban life experiences in a territory of social vulnerability. Methods: It was based on a qualitative approach, using focus group and semi-structured interview, applied to 45 participants. Content analysis was carried out, thus enabling the categorization of data with use of the thematic analysis technique. Results: The results showed the experiences of the participants in the territory with insufficient supply of services that determine the health of the population, pointing out relevant challenges such as low coverage in Primary Health Care and urban violence. The search for health care quality in the Unified Health System was presented as a resistance strategy. It was pointed out the need for appreciation in the value of the territory as a locus of care for the individuals, with intersectorial actions aimed at promoting the health of the population. Conclusion: The study recognizes specificities of the social determinants in the health of populations presenting life conditions permeated by inequities and exposes subsidies for elaboration of measures that contribute to overcoming such conditions, in order to generate social equity.
\end{abstract}

Descriptors: Social Determinants of Health; Unified Health System; Health Promotion.

Este artigo foi selecionado, corrigido e aprovado pela Comissão Científica do Fórum Internacional de Sistemas Universais de Saúde, seguindo suas normas e formatação. 


\section{RESUMEN}

Objetivo: Comprender la relación entre el instituido constitucional sobre los determinantes sociales de la salud y la vivencia urbana de un territorio con situación de vulnerabilidad social. Métodos: Basado en el abordaje cualitativo con la utilización de grupo focal y entrevista semi estructurada realizados con 45 participantes. El analisis de contenido ha posibilitado la categorización de los datos a partir de la tecnica del analisis temático. Resultados: Los resultados mostraron la vivencia del territorio con insuficiente oferta de servicios que determinan la salud de la población apuntando los desafíos marcantes con baja cobertura para la Atención Primaria de Salud y la violencia urbana. La búsqueda por la calidad de la asistencia en el Sistema Único de Salud se presentó como estrategia de resistencia. Ha sido apuntada la necesidad de valorización del territorio como lócus de cuidado de las personas con acciones intersectoriales para la promoción de la salud de la población. Conclusión: Se reconocen las especificidades de los determinantes sociales de la salud de poblaciones que presentan condiciones de vida permeadas por las desigualdades y se exponen subsidios para la elaboración de medidas que puedan contribuir para la superación de eses cuadros de modo que genere la equidad social.

Descriptores: Determinantes Sociales de la Salud; Sistema Único de Salud; Promoción de la Salud.

\section{INTRODUÇÃO}

No Brasil, o fortalecimento da construção de um sistema de saúde com acesso universal, integral e de qualidade ocorreu no mesmo período histórico que a luta pela redemocratização do país. Esse momento político, embasado no movimento de Reforma Sanitária Brasileira, é pautado numa mobilização reivindicatória alicerçada na necessidade popular de reconstruir uma estrutura normativa que atendesse as reais necessidades da população nas questões de saúde como direito de cidadania ${ }^{(1,2)}$. Neste cenário, partiu-se de uma compreensão holística do processo saúdedoença e da perspectiva de que o Sistema Único de Saúde (SUS) deveria integrar os sistemas universais de saúde.

No instituído constitucional brasileiro, o art. 196 traz que "a saúde é direito de todos e dever do Estado, garantido mediante políticas sociais e econômicas que visem à redução do risco de doença e de outros agravos e ao acesso universal e igualitário às ações e serviços de promoção, proteção e recuperação"(3). Após três décadas de criação do SUS, em meio ao debate mundial sobre a cobertura universal de saúde em contraposição à noção consolidada de sistemas universais de saúde ${ }^{(4,5)}$, evidencia-se ainda o desafio de trabalhar os determinantes sociais da saúde ${ }^{(6,7)}$ em sistemas universais.

Considera-se que, numa caracterização atual, a promoção da saúde deve ter como foco da ação sanitária os determinantes sobre a saúde, que incluem o próprio indivíduo e seu estilo de vida; as redes sociais e comunitárias; a oferta de serviços como o de saúde, educação, habitação e saneamento; além das condições socioeconômicas, culturais e ambientais. A atuação das pessoas na melhoria de sua qualidade de vida e saúde que, nos países desenvolvidos, tem como ênfase as mudanças comportamentais e as regulações sociais, deve priorizar os processos comunitários voltados para mudanças sociais na América Latina ${ }^{(8)}$.

Nessa linha de pensamento, as iniquidades sociais ganharam relevância na agenda pública global, com destaque para a mobilização gerada pela Organização Mundial de Saúde (OMS) ${ }^{(9)}$ para promover uma tomada de consciência sobre a importância dos determinantes sociais na situação de saúde de indivíduos e populações e sobre as necessidades de saúde por eles geradas.

Na direção apontada pela OMS, ocorreram a Conferência Mundial de Determinantes Sociais da Saúde e a Conferência das Nações Unidas sobre Desenvolvimento Sustentável/ Rio + 20, ambas no Rio de Janeiro, Brasil, respectivamente em 2011 e 2012, em que os estados membros se comprometeram a fortalecer os sistemas de saúde para que se ofereça acesso universal e equitativo à saúde ${ }^{(10,11)}$.

Este estudo foi desenvolvido a partir desses contextos e da consideração de que os determinantes interagem para afetar cumulativamente a carga de doenças de indivíduos e populações, estabelecendo desigualdades e disparidades de saúde em todo e dentro dos países ${ }^{(12)}$. $\mathrm{O}$ objetivo do presente estudo foi compreender a relação entre o instituído constitucional sobre os determinantes sociais da saúde e a vivência urbana num território em situação de vulnerabilidade social.

\section{MÉTODOS}

Trata-se de um estudo descritivo e exploratório, com abordagem metodológica qualitativa. Buscou-se compreender a relação entre o instituído constitucional sobre os determinantes sociais da saúde e a vivência urbana da região 
do Grande Bom Jardim (GBJ), nos anos 90 e 2000. Focou-se num estudo de caso da experiência brasileira de Estratégia Saúde da Família( ${ }^{(13)}$, por ser o serviço de saúde um determinante social ${ }^{(6,14)}$ e a Estratégia Saúde da Família (ESF) um modelo coletivo de atenção primária, com a peculiaridade de ser construído no âmbito de um sistema público universal.

É parte integrante de uma tese de doutorado sobre os Determinantes Sociais da Saúde (DSS), desenvolvida entre 2011 e 2015, no GBJ, região do município de Fortaleza, Ceará(15), constituída por cinco bairros: Bom Jardim, Canindezinho, Granja Lisboa, Granja Portugal e Siqueira, representando 8,3\% da população de Fortaleza, com concentração dos indicadores sociais e econômicos que apontam acentuado contexto de pobreza, exclusão e vulnerabilidade social ${ }^{(16)}$.

Para contribuir com a compreensão dessa abordagem, apresenta-se a seguir o modelo de determinantes sociais que embasa este estudo: (Figura 1)

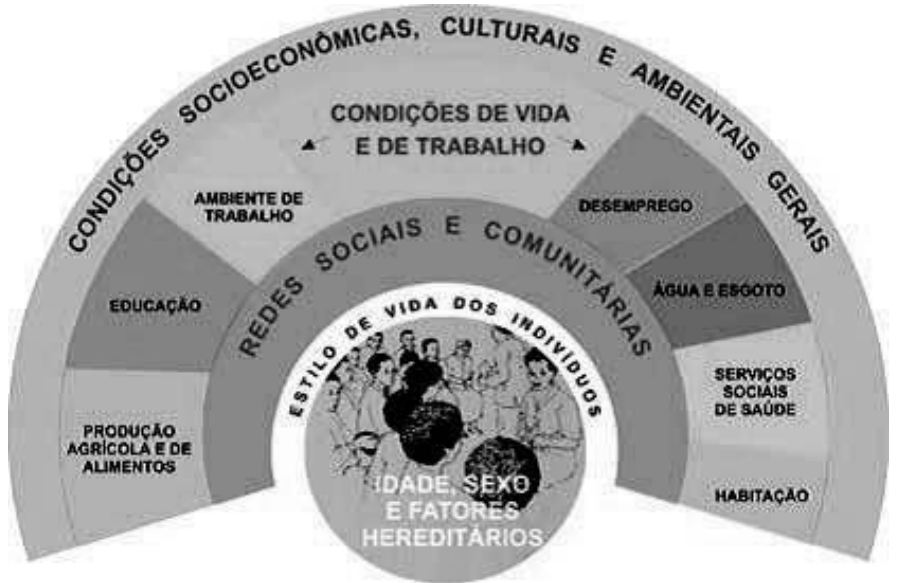

Figura 1- Modelo de Determinantes Sociais da Saúde de Dahlgren- Whitehead Fonte: Dahlgren e Whitehead, $1991^{(6)}$.

A região é caracteriza por uma infraestrutura precária e por uma grande demanda de serviços que propiciem melhores condições de vida à população. No mapa da extrema pobreza produzido pelo IPECE ${ }^{(17)}$, chama atenção o fato de quatro dos cinco bairros que compõem o GBJ estarem entre os dez de Fortaleza com o maior percentual de pessoas na extrema pobreza. Evidencia-se também a mobilização popular voltada para a exigibilidade dos direitos a serviços essenciais, como saúde e educação.

Os participantes foram 45 adultos inseridos no contexto de atuação social e política no GBJ, conforme Tabela I a seguir. Ao total, realizaram-se trinta e quatro entrevistas individuais e o grupo focal contou com a presença de onze pessoas que não participaram anteriormente de entrevistas. Para resguardar o anonimato dos participantes, seus nomes foram substituídos por outros fictícios e o grupo focal foi intitulado de Grupo Focal do Grande Bom Jardim (GFGBJ). Foram convidadas três entidades organizadas de cada bairro que compõem o GBJ. Quanto ao sexo, foram $20(44,4 \%)$ mulheres e $25(55,6 \%)$ homens. A idade dos participantes variou entre 25 e 78 anos.

Tabela I - Participantes da pesquisa. Fortaleza, Ceará, 2011-2015.

\begin{tabular}{lr}
\hline Participantes & $\mathbf{n}$ \\
\hline Líderes religiosos & 06 \\
Representantes de entidades organizadas (Associações Comunitárias e Organizações Não Governamentais - ONGs) & 15 \\
Trabalhadores da sede da Regional V - SER V (Educação, Saúde, Assistência Social, Infraestrutura e meio & 12 \\
ambiente, SINE) e trabalhadores do CRAS e do CAPS & 10 \\
Trabalhadores de unidades básicas de saúde & 02 \\
Vereadores & $\mathbf{4 5}$ \\
Total & 10 \\
\hline
\end{tabular}

SINE: Sistema Nacional de Emprego; CRAS: Centro de Referência de assistência Social; CAPS: Centro de Atenção Psicossocial

Como técnicas de coleta de dados, utilizaram-se a entrevista semi-estruturada e um grupo focal que durou $2 \mathrm{~h} 45 \mathrm{~min}$, que contou com onze participantes da pesquisa e um observador, por meio de perguntas disparadoras, 
como "O que vocês têm a dizer sobre a história das políticas públicas no GBJ? Há políticas públicas que não eram priorizadas e passaram a ser? Há políticas que não avançaram? Se sim, por quais motivos? Na realidade do GBJ, vocês acham que os serviços de saúde influenciam na saúde das pessoas?"

A partir dessas questões, os participantes discorreram sobre a evolução temporal de algumas políticas, dentre elas a saúde, e as possíveis influências delas na saúde da população local. Para análise do corpus de dados utilizouse a análise de Conteúdo ${ }^{(18)} \mathrm{com}$ a técnica Análise Temática e o auxílio do software Atlas Ti 5.2.

O trabalho recebeu aprovação de comitê de ética em pesquisa, conforme resolução $n^{\circ} 466 / 12$, com $n^{\circ}$ do processo: 26276413.7.0000.5040.

\section{RESULTADOS E DISCUSSÃO}

\section{Vivência no território: insuficiência na oferta de serviços que determinam a saúde da população}

O SUS constitui-se de um conjunto de redes de atenção à saúde articuladas com a finalidade de desenvolver atenção integral que exerça impacto na situação de saúde das famílias, indivíduos e comunidade e, também, nos determinantes e condicionante da saúde das coletividades ${ }^{(13)}$. Neste estudo, os participantes trouxeram reflexões sobre a saúde no cotidiano, apontando para uma compreensão na perspectiva da determinação social:

"Fazer saúde, pra mim, é pensar no ser humano no todo, ele está residindo numa área propícia? Ele tem escola próximo da casa dele pra que os filhos tenham acesso à educação? Ele tem uma unidade de saúde próxima a casa dele pra que os filhos dele, e ele também, possam ter acesso a um programa de saúde preventiva, não é? Qual é o trabalho que ele desenvolve? Qual é a oportunidade de trabalho que aquela pessoa tá tendo? Ai, ele está sem emprego, está sem condições, está sem rumo na vida, porque não o estado compreender que a obrigação é do estado, não é? (Aroldo, membro do Conselho Regional de Saúde da Regional SER V).

Uma forma de compreender saúde que abrange os serviços de saúde, mas também a oferta de serviços oriundos das políticas de educação, de habitação e de emprego e renda, responsabilizando o Estado pela implantação e implementação de políticas públicas que garantam o bem-estar da população, o que coaduna com as ideias da literatura(19):

"Eu acredito que às vezes as pessoas trabalham muito na perspectiva de doença e não de saúde também, não é, então, quando eu estou doente eu corro pra o hospital e não tenho a ideia de que o meu processo de vida vai me influenciar ou não na minha doença não é [...] e aí eu acho que até a própria estratégia saúde da familia ajudou também nesse processo de tentar mudar isso com as pessoas, entendeu?" (Vanessa, trabalhadora do CAPS).

No contexto da pesquisa, as pessoas trouxeram a compreensão de que a vida que se leva interfere na saúde, apontando também para o sistema de saúde como partícipe desse processo. Associa-se a isso a construção do SUS como uma iniciativa coletiva na consolidação de um modelo público de serviços para as pessoas no território, que intenciona reverter um modelo, de atenção à saúde, pautado na cura para uma perspectiva preventiva e promotora de saúde, priorizando as demandas do território consoante com os desafios do sistema ${ }^{(20)}$.

A ESF, como um modelo coletivo de Atenção Primária à Saúde (APS), foi apontada como contribuinte no acompanhamento das pessoas no seu processo de saúde e de vida. Isso representa a longitudinalidade, que juntamente com a acessibilidade, a integralidade e a coordenação do cuidado representam quatro elementos essenciais da APS ${ }^{(21)}$.

Dentro da proposta de fortalecimento da APS e implantação da ESF, a presença dos agentes comunitários de saúde (ACS) foi significativa no GBJ, local onde, segundo uma enfermeira entrevistada, "a gente vê muita doença, principalmente de pele, por conta de mosquitos e de coisas porque está tudo a céu aberto. É lama, é tudo" (Aparecida, trabalhadora da ESF).

Um dado que se destacou no local estudado foi o aumento do número de agentes comunitários de saúde no período de enfrentamento de epidemias. Entre as décadas de 1980 e 1990, duas doenças assolaram os cearenses: cólera e dengue, sendo esta última ainda motivo de preocupação na atualidade. O vibrião colérico encontrou terreno fértil no Brasil, devido à precariedade do saneamento básico, característica marcante também do GBJ, como descrito a seguir:

"Eu entrei em 92, na época daquela epidemia de cólera, não é. Ah, foi terrível! [...] esse posto aqui que eu estou [...] tinha umas macazinhas, uma em cima da outra, para poder comportar os pacientes que a gente trazia de 
dentro das comunidades [...] e a gente tinha que entrar mesmo na lama, a pé, e buscar o paciente lá, trazer no braço, tá entendendo, botar no carro de mão para poder ter acesso até a ambulância para trazer pro posto, porque não dava tempo. Como matava dentro de 24 horas, não dava tempo levar para o hospital [...] paciente se desidratava muito rápido e morria. Então, nessa época, morreu muita gente, não é, foi assim, foi uma coisa mais horrivel [...] no Canindezinho a epidemia foi maior porque na época não tinha água encanada, era água de cacimba, então os dejetos que todo mundo, que chovia e a água levava para lá e contaminava tudo, aí todo mundo ficava contaminado com a cólera." (Paula, trabalhadora de unidade básica de saúde do GBJ).

A história das pessoas e do lugar pesquisado é marcada por vivências de situações como essa, em que o processo saúde-doença se apresenta debilitado diante da precariedade das condições de vida, onde a falta de planejamento urbano evidencia-se até na largura da rua, que não propicia a passagem de uma ambulância. Os participantes apontaram o avanço nos serviços de saúde a partir da década de 1990 e essa epidemia de cólera foi um marco nesse sentido, pois apesar de estarem observando o alastramento da doença e a morte de muitas pessoas, perceberam uma "resposta governamental" no cuidado com a saúde das pessoas, que se expressou de formas diferenciadas.

Além da contratação de mais ACS para os serviços de saúde, foram citadas drenagens nas ruas, o aumento do número de ruas com calçamento, o que contribuiu para melhoria nos deslocamentos e diminuição da "poeira solta", que facilitavam os processos de adoecimento. Quanto ao saneamento, os dados do IBGE (2010)(22) ainda apontam cobertura inferior a $30 \%$ no GBJ, índice muito baixo, que indica a necessidade de melhorias, especialmente quanto ao esgotamento sanitário na capital cearense.

No setor saúde, a contratação de profissionais, a construção de mais uma unidade básica e a reforma de unidades nos anos 2000 foram dados destacados. Na contemporaneidade, são 08 unidades básicas de saúde, compondo um total de 20 unidades na SER V e 91 no município de Fortaleza ${ }^{(23)}$. Após concurso público nos anos 2000 , foram contratadas profissionais de nível superior para 300 equipes e a cobertura da ESF em Fortaleza passou de 15\% para $43,4 \%$ da população local. É preciso registrar a significância do avanço, no entanto, a cobertura não atingiu $50 \%$.

\section{Desafios marcantes para a cobertura adequada da ESF: poucos profissionais e a violência urbana}

As vagas disponíveis para atuação no sistema de saúde na região do GBJ não foram todas ocupadas mesmo após concurso público. Os participantes trouxeram que, quando um profissional sabe que será lotado no GBJ, pensa em desistir da vaga por motivos como a distância ao centro da cidade e, especialmente, a dificuldade de acesso e à violência presente nessa região da cidade e difundida na mídia cotidianamente.

Um aspecto relevante existente no GBJ que tem prejudicado o desenvolvimento de ações dos serviços de saúde é a violência urbana, considerada a maior causa de acidente de trabalho fatal no Brasil( ${ }^{(24)}$. Os participantes relataram casos em que os agentes comunitários de saúde e médicos foram assaltados em horário de serviço; viram-se no meio de tiroteio entre grupos de jovens, quando estavam se deslocando de uma rua para outra; realizavam visita domiciliar e a polícia chegou à casa, assustando os profissionais, especialmente pelo fato de temerem que os moradores considerem que eles foram os denunciantes dos ilícitos existentes no domicílio; enfermeiros que precisaram sair escoltados de uma comunidade. Esses fatos fazem com que profissionais temerosos não queiram mais trabalhar no GBJ e, mais ainda, cumprir os horários de funcionamento das unidades de saúde até $19 \mathrm{~h}$, o chamado terceiro turno, pois a frequência das ocorrências aumenta ao entardecer.

Um dos participantes trouxe que, devido ao reconhecimento desses elementos, a prefeitura de Fortaleza propôs uma gratificação para os profissionais que trabalhassem em áreas como o GBJ:

"A prefeitura criou a gratificação financeira pra profissionais que fossem pra áreas mais distantes do centro de Fortaleza, então, quanto mais distante, maior é a gratificação, pra atrair profissionais pra essas áreas, de todas as categorias: médico, enfermeiro, dentista, nível superior. Eu acho que isso foi um grande avanço." (Roberto, trabalhador da sede da SER V).

Apesar desses incrementos, parte dos profissionais concursados e lotados para trabalhar na SER V de Fortaleza não permaneceu nesses postos de trabalho, pelos motivos já mencionados. Para os entrevistados, soma-se a isso uma característica muito forte no GBJ, que é o constante aumento populacional e, portanto, diminuição proporcional da cobertura de saúde, que está entendida nesse estudo como acesso e uso do serviço(4):

"Nós, por exemplo, no Bom Jardim, devido toda essa montoeira de gente que inchou o Bom Jardim, os postos quebrados que nós temos, não é, e a falta de médico que a gente tem do programa saúde da família. OAbner, 
por exemplo, é em torno de cinquenta mil pessoas, não tem a mínima condição, se trabalha com isso, com essa ideia, que a demanda é essa, mas não se atende essa demanda, entendeu? Não atende porque não tem a mínima condição, então, todos os postos daqui são assim, todos, é independente, não tem nem melhor e nem pior, todos são iguais." (GFGBJ).

O dado trazido pela participante, de que há unidade básica de saúde no GBJ com responsabilidade sanitária de uma população de cerca de 50 mil pessoas, mesmo que não seja preciso, chama a atenção, pois denuncia o quanto está distante daquilo que é preconizado pelo Ministério da Saúde. De acordo com a portaria $n^{\circ} 2436 / G M$, de $2017^{(25)}$, que aprovou a Política Nacional de Atenção Básica e reviu as diretrizes para a organização da Atenção Básica, no âmbito do SUS, a Atenção Básica deverá ser ofertada integral e gratuitamente a todas as pessoas, de acordo com suas necessidades e demandas do território, considerando os determinantes e condicionantes de saúde.

O parâmetro para atingir o potencial resolutivo, considerando a população adscrita por equipe de dois a três mil e quinhentas pessoas, e quatro equipes por UBS, é de uma UBS para até 14 mil habitantes. Considerando que o GBJ tem 8 UBS, se fosse utilizado o parâmetro para até 14 mil habitantes em cada uma delas, daria para cumprir a responsabilidade sanitária com até 112 mil habitantes, o que representa cerca de $50 \%$ da população da região estudada.

Nesse contexto, é interessante mencionar também o quantitativo de ACS, que nem sempre é adequado. De acordo com a legislação em vigor, em áreas de risco e vulnerabilidade social, recomenda-se a cobertura de $100 \%$ da população, com um máximo de 750 pessoas por ACS. Conforme relatos, houve melhorias significativas quanto à isso, pois houve seleção para o emprego público de ACS, em meados dos anos 2000. No entanto, passada uma década, há ainda área descoberta em relação ao trabalho dos ACS também.

Além das situações descritas de áreas descobertas oficialmente, por questões relativas ao processo de chamado da seleção pública, há outras que se dão na informalidade e foram comentadas pelos participantes. Um exemplo foi a definição de uma Conjunto Habitacional, que tem 744 famílias, como uma microárea chamada pelos profissionais de saúde, em tom de brincadeira, de macroárea. Isso quer dizer que o ACS tem a responsabilidade de trabalhar com quase 750 famílias e não com 750 pessoas, como preconizado pela legislação em vigor. Ao total, são mais de 2500 pessoas no Conjunto Habitacional que, de acordo com o profissional entrevistado, recebem visita de $3 \mathrm{em} 3$ meses, o que faz, por exemplo, que uma gestante seja vista apenas umas 3 vezes por seu ACS, o que compromete a qualidade do serviço.

Esses elementos fazem com que a proposta da Estratégia Saúde da Família não seja implementada com todo vigor, a partir da preconização da equipe de caráter multiprofissional que trabalha com definição de território de abrangência, adscrição de clientela, cadastramento e acompanhamento da população residente na área ${ }^{(26)}$. Se os meios são alterados, começam as dificuldades também no alcance dos objetivos de desenvolver ações de promoção da saúde, prevenção de doenças, cura e reabilitação:

“[...] a saúde hoje acabou a questão da saúde da mulher, que antes tinha prevenção, não é, do câncer, hoje a gente não tem mais nos postos de saúde, pelo menos em alguns postos não tem; outra situação é a questão também da saúde do acompanhamento, não é, da questão dos tbs (pessoas com tuberculose) e Hansen." (Daniela, representante de entidade organizada).

Foram relatadas dificuldades do cumprimento, inclusive, dos programas de controle de algumas doenças, como visto anteriormente. Os participantes afirmaram que, até 2010, recebiam orientações, faziam palestras em associações e escolas, formavam grupos de adolescentes, tinham mais cuidado com o acompanhamento. Essas práticas integram a proposta de educação permanente em saúde, que, visando um maior poder de resolução, satisfação e adequação das ações e dos serviços propostos às necessidades de saúde da população, devem apresentar uma gama de variedade para que alcance diferentes públicos e, consequentemente, objetivos variados na saúde ${ }^{(27)}$.

No entanto, são observadas falhas não só no processo de educação em saúde, mas na implementação da própria ESF. Isso se revela em aspectos como a falta de estrutura para realização das visitas, a diminuição do fornecimento de carros para a realização de visitas em domicílios mais afastados da unidade básica; existência de unidades que estão há muitos anos sem fazer prevenção ginecológica; puérperas que ficam ser receber visita dos profissionais de saúde; ausência de material sobre as atividades das unidades, para que os agentes comunitários deem mais suporte informativo à comunidade. Dessa forma, fica claro o distanciamento entre o que está instituído na legislação em vigor e o que é vivido pela população de uma região periférica de grande cidade. 


\title{
Busca por qualidade da assistência: estratégia de resistência
}

A precarização da estrutura de unidades de saúde no GBJ foi comentada pelos participantes deste estudo. Numa fala sobre determinada unidade, teve-se que: "o teto estava para cair em cima do pessoal, o cupim tinha comido a madeira todinha, a qualquer hora podia desabar" (GFBJ). Além de outros casos comentados e de um que considerou-se ilustrativo em relação à necessidade de priorização da estrutura das unidades:

\begin{abstract}
"O posto Dom Lustosa, ele tem várias fossas assépticas assim do lado, tem um terreno e sempre está inundado de água da fossa, né, inclusive agora, esse mês agora teve essas chuvas muito e na entrada do posto tem um esgoto que estava jorrando água lá das fossas pra dentro do posto, não é, com dejetos fecais lá dentro do posto [...]." (GFGBJ).
\end{abstract}

Essa situação, de dejetos fecais entrando na unidade de saúde, demonstra a interligação entre vários aspectos que compõem o processo saúde-doença. Um deles é a falta de saneamento da região, interferindo na própria condição de funcionamento de uma unidade de saúde e, obviamente, na qualidade do atendimento. Em decorrência disso, embora a unidade tenha passado por reforma na gestão de 2009-2012(28), e de outras condições para o desenvolvimento do próprio trabalho, os profissionais de saúde relataram que eles e seus colegas se sentem muito desgastados no exercício do trabalho.

Um profissional chegou a relatar que um colega não atendeu um rapaz, que demandava a troca de curativo em seu pé, após já ter ido outra vez à unidade básica, caminhando 40 minutos de sua casa até o local. $O$ atendimento não foi realizado com a justificativa de que a sala onde o procedimento deveria ser feito estava com a lâmpada queimada. A reflexão proporcionada pela fala dessa participante é sobre a necessidade de fortalecer a Política Municipal de Humanização (PMH).

A PMH visa à humanização dos serviços e das ações de saúde, segundo os princípios do SUS ${ }^{(29)}$. Os relatos trouxeram a dimensão da necessidade de progresso quanto ao acolhimento e à integralidade do cuidado ${ }^{(30)}$, pois permanece uma sensação muito forte de insatisfação quanto ao trato no atendimento, que se revela em falas como esta: "o pessoal trata as pessoas como que a gente vai ali porque a gente quer, não é, gente, é por necessidade [...] a gente precisa de atendimento de qualidade, as pessoas necessitam ser atendidas como gente e não como bicho..." (GFGBJ).

Além disso, ficou clara a necessidade de que sejam revistas as condições de trabalho dos profissionais de saúde. Um dos participantes trouxe, por exemplo, que os usuários estavam fazendo muitas denúncias, no Conselho Regional de Saúde, contra um médico que trabalha numa unidade de saúde. Quando a unidade foi visitada, identificou-se que o médico estava priorizando os atendimentos de acordo com o agravo à saúde, isto é, fazendo o Acolhimento com Classificação de Risco - $\mathrm{ACCR}^{(31)}$, pois o número de usuários/dia que chegavam para ele correspondia à cerca de 100 pessoas. Para os participantes, atender a esse quantitativo é "humanamente impossível" (Pedro, trabalhador de unidade de saúde), o que resgata as condições e os limites de cada ator envolvido nesse processo.

A partir do ano de 2014, essas situações passaram por mudanças devido ao Programa Mais Médicos ${ }^{(32)}$, que pode permitir completar equipes de saúde da família quanto ao profissional médico: "E as equipes quase todas aqui, pelo menos, quando a gente fez aquele diagnóstico, no começo da gestão, era quase todas incompletas, agora com a vinda do Mais Médicos, não, a gente tem conseguido completar as equipes" (Antônio, representante de entidade organizada).

Esse diagnóstico referido pelo participante é fruto do trabalho de representantes de entidades do GBJ, que se reúnem e fazem visitas periódicas às unidades de saúde para acompanhar a situação dos serviços, o que vem apontando para o aumento do quantitativo de médicos nas unidades, também relacionada com a implantação de mecanismos para que esses profissionais, em geral, cumpram seus horários de trabalho.

Essa situação da área estudada mostra o esforço de superação da dificuldade de provimento dos serviços de saúde em regiões periféricas, que também tem relação com a inadequada distribuição geográfica de profissionais de saúde. Isso acontece pela ausência de atratividade de regiões com piores indicadores sociais, condições inadequadas de trabalho, cargas horárias excessivas e má remuneração, o que dificulta a fixação dos médicos em áreas remotas e/ou vulneráveis ${ }^{(33)}$.

Além disso, a construção de novas unidades de saúde no município não implicou, por exemplo, no aumento do número de equipes de saúde da família, com contratação de profissionais por concurso público. Há precarização do processo de trabalho e pessoas que vivem na área adscrita à unidade de saúde sem assistência assegurada. Concorda-se com a literatura( ${ }^{(34)}$ quando se afirma que ampliar o acesso e atenuar as desigualdades em saúde através da distribuição de profissionais na atenção primária exige ações de longo prazo, o Programa Mais Médicos 
pode ser uma iniciativa importante. No entanto, é preciso resistência no sentido de ampliação das estratégias que propiciem qualidade na assistência, como melhoria da infraestrutura, da distribuição de insumos e equipamentos e, especialmente, a garantia de condições para o desenvolvimento de ações intersetoriais, que possam resultar na prevenção de doenças e na promoção de saúde nos territórios, na perspectiva ampliada da determinação social.

\section{CONSIDERAÇÕES FINAIS}

O estudo trouxe à tona desafios e avanços na Estratégia Saúde da Família. Viu-se que o número de unidades básicas e de equipes de saúde da família aumentou, mas continua insuficiente no território analisado. A população expressa o aumento da expectativa de vida e da demanda aos serviços de saúde, mas não tem a vivência da garantia ao acesso universal.

A região estudada caracteriza-se pela situação de vulnerabilidade social e contempla uma população que se utiliza quase na sua totalidade do serviço público de saúde. Melhorar a oferta de serviço de saúde nessa região, quantitativa e qualitativamente, seria, portanto, uma forma importante de reduzir desigualdades sociais no município.

Apesar da clareza sobre a importância da implementação de políticas públicas que garantam a eficácia e a eficiência do sistema de saúde, a perspectiva dos determinantes sociais mostra que os modelos assistenciais de cuidado em saúde não são suficientes para efetivamente melhorar a saúde individual e da população e promover a equidade em saúde. Constatou-se, por exemplo, a (in) segurança pública como um determinante social que tem grande influência negativa na saúde da população do Grande Bom Jardim. Isso aponta para a necessidade de valorização do território como lócus de cuidado das pessoas, com ações intersetoriais que visem prevenir a violência e promover a saúde da população.

A situação é complexa e exige o reconhecimento das especificidades dos determinantes sociais da saúde de populações que apresentam condições de vida permeadas por iniquidades, as quais precisam ser consideradas para que se tenham subsídios para elaborar medidas que contribuam para a superação de quadros como esse, de forma a gerar equidade social.

Fonte financiadora da pesquisa: Coordenação de Pessoal de Aperfeiçoamento de Nível Superior (CAPES).

Artigo baseado na Tese: Os Determinantes Sociais da Saúde no Grande Bom Jardim - Fortaleza (CE), Universidade Federal do Ceará, 2015, 380f.

\section{REFERÊNCIAS}

1. Paim JS. Reforma Sanitária Brasileira: contribuição para a compreensão crítica. Rio de Janeiro: Fiocruz; 2008.

2. Machado MFAS, Monteiro EMLM, Queiroz DT, Vieira NFC, Barroso MGT. Integralidade, formação de saúde, educação em saúde e as propostas do SUS: uma revisão conceitual. Ciênc Saúde Colet. 2007;12(2):335-42.

3. Brasil. Constituição da República Federativa do Brasil: promulgada em 5 de outubro de 1988. São Paulo: Saraiva; 1988.

4. Noronha JC. Cobertura universal de saúde: como misturar conceitos, confundir objetivos, abandonar princípios. Cad Saúde Pública. 2013;29(5):847-49.

5. Rodin J, Ferranti D. Universal health coverage: the third global health transition? Lancet. 2012;380(9845):861-2.

6. Dahlgren G, Whitehead M. Policies and strategies to promote social equity in health. Stockholm: Institute of Futures Studies; 1991.

7. Dahlgren G, Whitehead M. Policies and strategies to promote social equity in health. 2a. ed. Background document to WHO - Strategy paper for Europe. Stockholm: Institute of Futures Studies; 2007.

8. Carvalho Al, Buss PM. Determinantes sociais na saúde, na doença e na intervenção. In: Giovanella L. Políticas e sistema de saúde no Brasil. Rio de Janeiro: Fiocruz; 2012.

9. World Health Organization. Commission on Social Determinants of Health (CSDH). A conceptual framework for action on social determinants of health [Internet]. 2007; [acesso em 2018 Fev 10]. Disponível em: www.who.int/ social_determinants/resources/latest_publications/en/index.html. 
10. Organização Mundial de Saúde. Declaração Politica do Rio de Janeiro sobre Determinantes Sociais da Saúde [Internet]. 2011; [acesso 2015 Dez 15]. Disponível em: http://www.who.int/sdhconference/declaration/ Rio_political_declaration_portuguese.pdf.

11. Organização das Nações Unidas. El futuro que queremos. Resolución aprobada por la Asamblea General el 27 de julio de 2012 [Internet]. 2012; [acesso 2018 Fev 09]. Disponível em: http://daccess-dds-ny.un.org/doc/ UNDOC/GEN/N11/476/13/PDF/N1147613.pdf?OpenElement

12. Andrade LO, Pelegrini A Filho, Solar O, Rígoli F, Salazar LM, Serrate PC; et al. Social determinants of health, universal health coverage, and sustainable development: case studies from Latin American countries. Lancet. 2014;385(9975):1343-51.

13. Andrade LOM, Barreto ICHC, Ribeiro KG, Uchoa AAC. A Estratégia saúde da família e o SUS. In: Rouquayrol MZ, Gurgel M, organizadores. Epidemiologia \& Saúde. Rio de Janeiro: Medbook; 2018.

14. Ribeiro KG, Barreto ICHC, Aguiar JB, Andrade LOM. Determinantes sociais da saúde. In: Rouquayrol MZ, Gurgel M, organizadores. Epidemiologia \& Saúde. Rio de Janeiro: Medbook; 2018.

15. Ribeiro KG. Os Determinantes sociais da saúde no Grande Bom Jardim - Fortaleza (CE) [tese]. Fortaleza: Universidade Federal do Ceará; 2015.

16. Carlos CAF. Invenções Democráticas pela Periferia: a rede de desenvolvimento local, integrado e sustentável do GBJ [dissertação]. Fortaleza: Universidade Federal do Ceará; 2014.

17. Instituto de Pesquisa e Estratégia Econômica do Ceará. Informe 44: perfil municipal de Fortaleza. Fortaleza: IPECE; 2012.

18. Bardin L. Análise de Conteúdo. São Paulo: Edições 70; 2014.

19. Buss PM, Pellegrini A Filho, Espiridião MA. Promoção da saúde e seus fundamentos: determinantes sociais de saúde, ação intersetorial e políticas públicas saudáveis. In: Paim JS, Almeida N Filho, organizadores. Saúde coletiva: teoria e prática. Rio de Janeiro: MedBook; 2014. p. 305-26.

20. Pinto AGA, Jorge MSB, Marinho MNASB, Vidal ECF, Aquino PS, Vidal ECF. Experiences in the family health strategy: demands and vulnerabilities in the territory. Rev Bras Enferm. 2017;70(5):920-7.

21. Starfield B. Atenção Primária: equilíbrio entre necessidades de saúde, serviços e tecnologia. Brasília: Ministério da Saúde; 2002.

22. Instituto Brasileiro de Geografia e Estatística. Censo Demográfico 2010 [Internet]. [acesso em 2018 Fev 08]. Disponível em: http://www.sidra.ibge.gov.br/cd/cd2010rpu.asp?o=6\&i=P

23. Fortaleza. Prefeitura Municipal. Secretaria Municipal de Saúde. Unidade de Saúde V (SMS). Postos. Regional I, II, III, IV, V, VI. 2015; [acesso em 2015 Mar 08]. Disponível em: http://www.fortaleza.ce.gov.br/sms/regional

24. Cordeiro R, Luz VG, Hennington EA, Martins ACA, Tófoli LF. A violência urbana é a maior causa de acidente de trabalho fatal no Brasil. Rev Saúde Pública. 2017;51:123.

25. Brasil. Ministério da Saúde. Portaria n. 2.436, de 21 de setembro de 2017. Aprova a Política Nacional de Atenção Básica, estabelecendo a revisão de diretrizes para a organização da Atenção Básica, no âmbito do Sistema Único de Saúde (SUS). Diário Oficial da República Federativa do Brasil, Brasília. 2017.

26. Almeida PF, Giovanella L, Mendonça MHM, Escorel S. Desafios à coordenação dos cuidados em saúde: estratégias de integração entre níveis assistenciais em grandes centros urbanos. Cad Saúde Pública. 2010;26(2):286-98.

27. Farias QLT, Rocha SP, Cavalcante ASP, Diniz JL, Ponte OA Neto, Vasconcelos MIO. Implicações das tecnologias de informação e comunicação no processo de educação permanente em saúde. RECIIS. 2017;11(4):1-11.

28. Rede de Desenvolvimento Sustentável do Grande Bom Jardim. Comissão de Segurança Humana. Informe local de violação aos direitos humanos: o direito à saúde no Grande Bom Jardim. Fortaleza: Centro Herbert de Souza, Escola Popular de Educação em Direitos Humanos; 2013.

29. Fortaleza. Prefeitura Municipal. Secretaria Municipal de Saúde. Relatório de Gestão do ano de 2008 da Secretaria Municipal de Fortaleza: saúde, qualidade de vida e a ética do cuidado. Fortaleza: SMS; 2009.

30. Rodrigues MP, Melo RHV, Vilar RLA, Silva GSN, Silva AB. Ressignificando o trabalho na estratégia saúde da 
família: desafios para a integralidade do cuidado em saúde. Revista Brasileira de Inovação Tecnológica em Saúde. 2017;7(2):32-44.

31. Brasil. Ministério da Saúde. Núcleo Técnico da Política Nacional de Humanização Humaniza SUS. Acolhimento com avaliação e classificação de risco: um paradigma ético-estético no fazer em saúde. Brasília: Ministério da Saúde; 2004.

32. Brasil. Lei 12.871, de 22 de outubro de 2013. Institui o Programa Mais Médicos, altera as Leis 8.745, de 9 de dezembro de 1993, e 6.932, de 7 de julho de 1981, e dá outras providências. Diário Oficial da República Federativa do Brasil, Brasília. 2013.

33. Scheffer M. Programa Mais Médicos: em busca de respostas satisfatórias. Interface Comun Saúde Educ. 2015;19(54):637-40.

34. Martins ACP, Sant'anna PA, Rocha JFD, Vieira MA. Mais médicos: uma revisão integrativa. Rev Bras Promoç Saúde. 2017;30(4):1-14.

\section{Endereço para correspondência:}

Kelen Gomes Ribeiro

Faculdade de Medicina - Universidade Federal do Ceará - UFC

Rua Prof. Costa Mendes, $1608 / 5^{\circ}$ Andar

Bairro: Rodolfo Teófilo

CEP: 60430-140 - Fortaleza - CE - Brasil

E-mail: kelengomesr@yahoo.com.br 